\title{
Un apocalipsis perverso: reflexiones en torno a la violencia simbólica en Eloy
}

\section{Resumen}

El artículo propone una lectura de la violencia simbólica en la novela Eloy (1960), del escritor chileno Carlos Droguett. Se comienza analizando el vínculo entre Eloy y las mujeres para presentar la estructura psíquica de la perversión. Luego se aborda la violencia simbólica a la luz de la teoría de René Girard sobre el motivo literario de los hermanos. Habría dos dobles de Eloy: el policía y el barbero. La relación de ambos con el bandido rural se caracteriza por su naturaleza perversa, que funciona del mismo modo: la violencia es caracterizada como violencia estatal, contenida y secular, que denuncia la existencia de un lazo social reificado entre campo y ciudad. Se termina ensayando una explicación para este doble uso de la perversión desde el contexto de producción de la novela.

Palabras claves

Carlos Droguett, Eloy, violencia simbólica, lazo social, perversión

\begin{abstract}
This essay approaches the symbolic violence in Carlos Droguett's novel Eloy (1960), opening with an analysis of the link between Eloy and women in order to present perversion as a psychic structure. The essay then addresses symbolic violence with reference to René Girard's theory concerning the literary motif of the brothers; it proposes there would be two doubles of Eloy: the policeman and the barber. The relationship between both of these figures and the main character (a rural bandit) is characterized by its perverse nature, whereby violence is understood as state violence, restrained and secular, which reveals the existence of a reified social link between the countryside and the city. Finally, the work seeks to account for this double use of perversion by considering the novel's production context.
\end{abstract}

Keywords

Carlos Droguett, Eloy, symbolic violence, social links, perversion

Desde su aparición en 1960 y hasta nuestros días, Eloy es una de las novelas de Carlos Droguett ${ }^{1}$ que más interés suscita por parte de la crítica. Si bien

${ }^{1}$ Carlos Droguett (1912-1996) es un escritor chileno que la crítica ha ligado a la generación de 1938. Buena parte de su vida se desempeñó como periodista de diversos periódicos capitalinos, en los cuales plasmó un estilo cáustico y un compromiso político sin concesiones. Admirador de Salvador Allende y entusiasta de la Revolución Cubana, salió al exilio rumbo a Suiza en septiembre 
en sus relatos anteriores se reconocen inquietudes sociales y novedades técnicas, en este texto encontramos una representación radicalmente nueva de los marginados, ejecutada con un notable balance entre pulcritud y vehemencia. Tales méritos se suman al reconocimiento internacional que obtuvo la obra, finalista del Premio Novela Breve Seix-Barral, en 1959. La técnica narrativa de Eloy, basada sobre todo en la corriente de la consciencia, corroboró con nuevos bríos el quiebre fundamental con el criollismo que Manuel Rojas había iniciado con Hijo de Ladrón (1951), y lo hizo apropiándose justamente de uno de los personajes predilectos de los novelistas de la tierra: el bandido rural. Así, dejó atrás la mirada paternalista que condenaba al sujeto popular a una pasividad definida de antemano, pues al ubicarlo en un pasado cerrado, cancelaba su carácter histórico.

Dos de los aspectos centrales de la narrativa de Droguett son la violencia (estructural, social, simbólica) y la cristología, que confluyen en un proyecto escriturario que busca contar la historia no oficial de Chile, esa historia -social"desde abajo": "Traté de trabajar entonces con las dos manos para no perder detalle ni hilo, para recoger toda la sangre, para construirla otra vez, y que corriera más abundante por los cauces de nuestra historia" (Droguett, Los asesinados 8). En este sentido, sobre Eloy (1960) se ha dicho que escenifica una violencia apocalíptica, por cuanto se le atribuye al desastre postrero de la novela un valor positivo y redentor (Sicard 195) ${ }^{2}$. En este ensayo, transitaremos por una vía alternativa a aquella, entendiendo de modo diferente la relación entre sufrimiento y reivindicación social. Nuestra hipótesis, en breve, es que Droguett manipula el modelo alegórico del apocalipsis en clave perversa para expresar la

de 1975. Su aporte a la narrativa chilena y latinoamericana le valió el Premio Municipal de Santiago en 1953 y el Premio Nacional de Literatura en 1970.

${ }^{2}$ Una lectura similar, que trabaja sobre la relación entre alegoría e historia, pero de la obra de Droguett, se puede encontrar en Mauricio Ostria. "Carlos Droguett: La novela como interpretación histórica". Acta Literaria, 20, 1995: 55-64. Tal lectura se basa en los supuestos hermenéuticos de la noción de figura, que acuñara Eric Auerbach. Numerosa evidencia textual avala esta propuesta: la figura de Cristo en el caballo/ Eloy rumbo al campo; la prostituta de Babilonia/ el pasado de Rosa; y, sobre todo, la unidad que bandido y naturaleza constituyen líricamente hacia el final del relato, en la forma de un diluvio con tintes apocalípticos. 
fracasada purgación del problemático vínculo social entre campo y ciudad, que caracteriza al Chile de la década de los cincuenta.

Eloy narra la historia de un "zapatero de extramuros" $(33)^{3}$ capitalinos que repentinamente experimenta una revelación y se interna en el campo, desde donde comienza su prontuario como bandido rural. El texto relata entonces su dilatada persecución por parte de los policías hasta su ajusticiamiento, mediante la técnica de la corriente de la conciencia, que monta un juego narrativo entre un punto de vista omnisciente y los oscilantes pensamientos del acosado. Pronto en la lectura se advierte que las mujeres juegan un papel central en la caracterización del personaje $\mathrm{e}^{4}$. Cuando comprende que lo persiguen, Eloy se da a la fuga, pero antes le dice a su pareja, Rosa, que lo espere junto a una botica. Buscando refugio, llega más tarde hasta un rancho donde encuentra a una mujer con su hijo pequeño. En adelante, la consciencia del criminal volverá una y otra vez sobre estas dos mujeres, ligadas no solo al deseo sexual, sino también a la figura de la madre. Es lo que sucede cuando, en plena persecución, el bandido bebe la leche contenida en el canasto de la mujer del rancho: "Bebió otro sorbo de leche y comprendió que la humilde y rotosa botella, con su amor humilde como su humilde entusiasmo, lo estaba uniendo invisiblemente a la mujer" (102); o en las identificaciones con niños, como cuando Toño, su hijo, juega con las balas de su padre, después de lo cual fantasea con los siguientes pensamientos de Rosa: "me estás mirando chorreado de sangre, estás viendo que los tiras me van a carnear, eso crees o esperas" (9). Destaquemos que la leche bebida acaba descomponiendo el estómago de Eloy, quien se la figura entonces envenenada, y que la segunda cita echa luz sobre la economía libidinal que dispone la novela. Todo ello nos alerta

\footnotetext{
${ }^{3}$ En adelante, todas las citas a Droguett corresponden a la novela que analizamos.

${ }^{4}$ El primer retrato que se hace del bandido es el siguiente: "un hombre cabal que en las ropas mezcladas y viajadas de las mujeres, en los calzones, las camisas de dormir, las zapatillas de levantarse bostezando y las medias de seda imperceptible y los encajes y los perfumes, los polvos y coloretes y pinturas al aceite o al petróleo, un hombre que ha pasado toda su vida barajando y revolviendo unas piernas, unos pechos de mujer" (14-15). En este pasaje, Eloy aparece entre los vestidos, prendas y demás objetos de las mujeres que tienen la función de adornar y cubrir sus cuerpos.
} 
de una relación de las mujeres con la fatalidad, representada en lo que el protagonista llama su destino: "él pensaba en la Rosa y le admiraba las piernas paradas en medio del sol, ella y ellas lo estaban esperando sin preocuparse ni cuidarse de nada más ni de dejar hacerlo, pues marcaban y eran su destino" (66).

El destino referido puede ser interpretado en términos de una fuerza pulsional que lo insta a actuar siguiendo el impulso de la sangre, que debe ser derramada. Como vemos, tal destino se encuentra ligado a las mujeres y, en definitiva, a un mandato de otro ante el cual el protagonista se muestra pasivo. Así, esta asociación delata la posición de sujeto que ocupa Eloy respecto de su sino: "the pervert's 'subject position' does not entail something outside or beyond the Other. Instead the pervert, as subject, plays the role of object: the object that fills the void in the mOther" (Fink 49). No es extraño entonces que el criminal muera entre violetas 5 , habiendo sostenido antes que "No hay flor más mujer que la violeta" (35), valoración que también explica que las balas caigan sobre el criminal con suavidad, "agradablemente perfumadas o narcotizadas, como si hubieran pasado todo ese tiempo y esas noches guardadas entre los pechos tibios y diminutos de la Rosa" (187). Lo que se suspende aquí es una lectura apocalíptica de la novela, según la cual el sujeto es identificado con una naturaleza bravía que azota la ciudad babélica y que anuncia su redención futura (ver nota al pie 2). Sin embargo, este no es el único modo en que se presenta la perversión en el relato.

El oficio de Eloy es dar la muerte a sujetos al margen de la ley; no obstante, su trabajo es equiparado al de otros asesinos, que operan al interior de aquella, y con quienes establece una relación de doble. Uno de ellos es el policía: en efecto, el acto por el cual el bandido adquiere tal condición es el asesinato de un carabinero, cuando revelación y muerte se expresan simultáneamente para indicarle un patrón de acción delictual. Acerca de otro uniformado, reflexiona:

\footnotetext{
5 “El olor de las violetas se le amontonó en la cara, subía por su mano hundida en el agua y se agarraba al barro y a su ropa, nunca había sentido tan eterno, leal, noble, franco y persistente el perfume delgado y humilde de las violetas" (190).
} 
"somos hace tiempo hermanos de sangre y de leche, colegas, compadres, socios señalados, yo mato y asesino fuera de la ley y usté al lado adentro de ella" (95). Hemos señalado que la calidad de doble se explica, a primera vista, por la relación con la ley y la muerte que ambos mantienen; en este sentido, el bandido rural es el contrapunto del policía. Es posible explicar este vínculo fraterno entre ambos a la luz de la noción de sacrificio, puesto que una lectura apocalíptica establecería que el policía es el sacrificador de Eloy. Como apunta René Girard, el motivo de los hermanos o dobles en la literatura muchas veces revela la existencia de un sacrificio que se realiza con el fin de asegurar el bienestar de la comunidad toda (49), pues esta ha caído en una crisis que precipita una violencia indiferenciada sobre el cuerpo social (punto que retomaremos hacia el final). En la novela, la crisis sacrificial estaría disfrazada por los desastres naturales y colectivos, y el modo de resolverla se basaría en la existencia de una "víctima propiciatoria": un elemento (animal o humano) dispensable que la sociedad acuerda en ultimar, acto que asegura su redención y refundación futura $(88,94-95)$. En definitiva, la religión se encargaría de sacralizar la violencia para entonces poder administrarla de modo preventivo; de ahí que "[...] el sacrificio, en el mejor de los casos, deb[a] ser definido como violencia purificadora" (47-48).

Ahora bien, hay un segundo personaje, protagonista de una escena clave de la novela, que también se presenta como hermano de Eloy: el peluquero. Recién iniciada la persecución, el bandido ingresa a una peluquería buscando tranquilidad. Se dice del dependiente que "son lo mismo que los pacos, los detectives y los matarifes, solo buscan el lugar más apropiado donde espolvorearte la pólvora, la navaja o el tajo" (64); "Es el peluquero y me dice que me sosiegue, podría ser un tira o un milico y también me diría que me sosegara" (65). En primer término, se describe un gesto mercantil: "tendió la cabeza hacia atrás y se dio cuenta de que el peluquero lo estaba tasando y apreciando con ojeada comercial y técnica, demoledora y definitiva, porque había adquirido su cabeza por media hora" (63); y un poco más adelante: “el peluquero no es un ser normal sino un primario y frustrado asesino, por eso, porque no se atreve, si se 
atreviera y se saliera de su amarra y su prohibición, desfilaría uniformado y condecorado por la muerte" (63). El barbero-hermano es un esteta de la muerte, un comerciante que maneja la violencia de modo distinto que el bandido, puesto que la contiene, la regula. En esta escena encontramos una prefiguración de la muerte final del protagonista. El sacrificio es -tan solo- insinuado del siguiente modo: "sentía los dedos del peluquero dibujarle una rayita de jabón en el bigote e inmediatamente [...] el fulano estaba furioso y asesino [...] Comprendió que lo había cortado" (66). Se trata de un corte leve, ciertamente no el que Eloy fantasea que ocurra, pues el perverso quiere que la ley caiga sobre sí plenamente, que lo avasalle; el bandido denuesta al barbero porque impide que ello suceda. Este sacrificio parcial corrobora la condición de perverso del bandido, sobre quien la ley no cae con todo su rigor, y quien por lo tanto ha sufrido una simbolización fallida. Como plantea Fink, la simbolización ineficaz, incompleta, implica que el niño no es capaz de vivir la separación respecto de la madre, con lo que continúa funcionando como un objeto de ella, tal como lo revisamos en las relaciones entre Eloy y las mujeres ${ }^{6}$. No obstante, la fantasía de Eloy presenta al barbero como asesino. ¿Cómo explicar esta transformación?

El peluquero manipula la cabeza de Eloy, la adorna, tal como Rosa se adorna a sí misma y a Eloy, quien está vestido como "pije"7 . Es coherente que precisamente en la peluquería el protagonista repare en su vestimenta, en esa camisa y corbata verde que su mujer le obsequiara, alentada por "sus amigas modocitas y decentitas" (60). Entendemos que la actividad ornamental disimula una condición oculta del protagonista, que aflora sobre la lavaza ensangrentada del barbero. Se nos dice que en la mirada de este último se halla la del rico citadino y la del conformista burgués (64). Además, tasa la cabeza del cliente para exponerlo humillantemente, como un maniquí, en el escaparate de la peluquería

${ }^{6}$ Cuando la simbolización es eficaz, el individuo es un sujeto deseante de la madre, y no un objeto de goce de aquella. Por esta razón, "perversion can be understood as owing to the absence or failure of symbolization" (Fink 53).

${ }^{7}$ Antes de entrar a la peluquería, el bandido piensa: “¿dónde te cortaste ceñido y pije el pelo?" (58). 
$(64)^{8}$. El delincuente, en tanto, presenta el "típico cogote de obrero de la zona central, obrero explotado y sin sindicato, de trabajador a trato del campo" (64). Podría argumentarse que Eloy desea que el barbero lo agreda, pues de ese modo se humanizaría el lazo entre ambos: el bandido sería reconocido como sujeto y se conectaría con el Otro real (Zizek 116). Efectivamente, en su fantasía el barbero busca violentarlo -"la navaja buscaba herirlo sin reparo, herirlo de nuevo, ya había olido su sangre y la averiguaba enloquecida" (67) - porque "soy de repente un pobre huaso campesino de la hacienda, el fundo o la parcela, felizmente para él ahora nada más soy un don nadie, rural, analfabeto e inerme [...] el campesino expropiado, el infeliz huaso expoliado" (67-68). En términos sociales, lo que se denuncia es una relación sádica: hay una dominación reificadora que el sujeto rural sufre a costa del burgués, y lo que Eloy fantasea perversamente es que tal vínculo se vuelva masoquista, es decir, que el barbero descargue sobre él su deseo, que es agredirlo furiosamente ${ }^{9}$. La violencia apocalíptica, que esta vez habilitaría el barbero, se encuentra nuevamente suspendida por el teatro que estetiza la violencia, aspecto que se vincula también con la relación perversa que Eloy mantiene con las mujeres.

Para caracterizar de forma completa la forma en que la perversión opera en la novela debemos atender también a la escena de acribillamiento del bandido, perpetuada por los policías. En las postrimerías de su agonía, Eloy siente compasión por uno de ellos que, enfermo como él mismo, tose. El narrador le cede la palabra para que se dirija al protagonista, le cuente sus penurias íntimas y, por último, uno y otro se identifiquen:

\footnotetext{
${ }^{8}$ Jaime Concha ha notado la obsesión de Droguett con los modos de intervenir el cuerpo y los diversos grados de invasión anatómica que aquellos suponen (Concha 120-121). En la novela que comentamos, el oficio del barbero revela un tratamiento de la violencia que sirve para encubrir estéticamente el aparato del dolor, al tiempo que inscribe el cuerpo del sujeto en la órbita mercantil: el pescuezo de Eloy se vuelve, en efecto, una mercancía.

${ }^{9}$ Sobre el lazo social masoquista, véase, Slavoj Zizek. "The Ambiguity of the Masochist Social Link”. Molly Anne Rothenberg, Dennis Foster y Slavoj Zizek (eds.). Perversion and the Social Relation. Durham/London: Duke University Press, 2003. 112-125.
} 
mi escritorio está lleno de sangre, esta tierra, este campo, este pasto, son mi escritorio, estoy trabajando, soy como tú, Eloy, igual que tú, nos parecemos [...] tenemos muchos papeles, listas de vivos, listas de muertos, listas de vivos que hay que matar, listas de muertos, nosotros los matamos, éstas son nuestras labores diarias, el orden normal de mi trabajo, aquí están mis vacaciones, mi desahucio, mi jubilación. (163)

Si bien la cita amarra la relación de dobles, permite por otro lado entender la diferencia entre los modos de violencia que los oficios de bandido y policía suponen. Las listas, la jubilación, el escritorio, nos remiten a la noción del trabajo asalariado estatal propio del carabinero, quien detenta una violencia que debe su legitimidad al Estado. Esta se caracteriza por su carácter rutinario, impersonal y regulatorio, propio del sistema judicial, con el funcionario estatal como paradigma de su subjetividad -y nótese que el vínculo entre barbero y policía también se desarrolla a nivel espacial, pues la peluquería es "salón y oficina" (70). Así, no es extraño que cuando le disparan, Eloy escuche que "se cruzaban voces de una oficina a otra, de una silla a otra, organizando otro poco de burocracia, sentía sonar claramente los fierros de las carabinas y las máquinas de escribir, el chirriar de la cortina metálica" (168). En la novela, la violencia de Estado no cuenta con la legitimidad suficiente como para instituir un sacrificio ya que es violencia sin más; el carácter técnico -ausencia de ritual- que la distingue impide entenderla como violencia sacrificial y, por tanto, redentora.

Por último, la reificación también hace su entrada en escena: los carabineros le disparan "como si no fuera un ser humano sino una cancha especial y reservada de tiro al blanco y, según las leyes y el orden establecido, no debiera moverse [...] tenía una piel y un destino no más, le disparaban pero no lo herían, ya no lo podrían herir nunca más, estaba saturado y cumplido su trabajo y su contrato, su sino y su estrella" (184, cursivas nuestras). Esta cita, nuevamente, contrasta con la fantasía de Eloy, quien piensa: "tienen que partirme y triturarme como a los bueyes y caballos en el matadero, por eso vinieron tantos, porque yo 
también soy numeroso" (164). Como con el barbero, el bandido reclama la necesidad de un ajusticiamiento bestial. Leída simbólicamente, la muerte del sujeto sería funcional al objetivo preventivo característico del sistema sacrificial, pues con el sacrificio de uno de los hermanos se aseguraría la supervivencia de la comunidad. La perversión, en tanto, se da por cuanto el sujeto debe presentarse pasivo frente al advenimiento de la ley sobre sí; en tal ecuación se mezcla la noción de que el individuo es un medio a través del cual se cumple un mandato que Otro impone, así como el goce reflejo que el perverso habilita en ese Otro, quien entonces se vuelve el sujeto de la pulsión (Lacan, citado en Penney 17): "los matorrales eran sacudidos por las balas [...] y él retrocedía apegado a las flores y los matorrales, experimentando una nueva y rara alegría, una loca seguridad y serenidad que ya conocía [...] una fiereza fría que le anunciaba un calor" (168).

Las violencias que tanto peluquero como policía escenifican convergen en una narrativa mayor, la de la violencia estatal. Por ello, la hermandad que los une a Eloy se circunscribe a las posiciones que uno y otros detentan respecto de la ley, pero esta es a la vez determinada por el tipo de administración de la violencia. Hemos visto que la mujer también juega un rol en esta relación, dado por la disimulación de esa violencia redentora con que Eloy fantaseara, la que sin embargo queda reducida a una vuelta hacia la madre, hacia las violetas. Nuestro énfasis en la negatividad - $\mathrm{O}$ en la falla- del apocalipsis no cancela una lectura política de la novela, sino que permite entender la ruina a que dicha alegoría de redención social fue reducida tras los avatares históricos que marcan la relación entre campo y ciudad en Chile.

Que el sacrificio del bandido no indique una salida positiva para la salvación del pueblo (la refundación futura de que habla Girard) podría dar cuenta del dilatado proceso de elaboración de la novela, problema hasta ahora omitido por la crítica. Aunque la primera versión del texto data de 1954 -y es publicada en 1960-, es recién en 1982 que Droguett establece una edición definitiva, escrita en Wabern, Suiza, con siete años de exilio a sus espaldas y con el beneficio de haber 
visto ya la decantación del proceso que se había iniciado con la Reforma Agraria. ¿Cómo explicarse este lapso de 28 años en que el bandido seguía merodeando por la mente del autor? El Estado, cuya implicación en cierta modalidad de violencia hemos revisado más arriba, nos puede dar una pista.

Las condiciones de producción de la novela superponen dos escenarios distintos: el caracterizado por un Estado de corte nacional-desarrollista ${ }^{10}$ y luego la dictadura militar (1973-1989), en que el neoliberalismo hace su entrada. En la década de los cincuenta, aún está vigente el primer escenario, en que los actores sociales (industriales, terratenientes, proletariado urbano) negocian acuerdos para asegurar la gobernabilidad nacional. Sin embargo, dicha estabilidad dependía en buena medida de la explotación campesina (Bauer 197); en efecto, tanto en los gobiernos radicales como en el de Ibáñez no se avanza en sindicalización campesina, y en cambio se perpetúa el sistema hacendal y las relaciones laborales paternalistas y autoritarias de los patrones, con el beneplácito eclesiástico. Los trabajadores urbanos, en cambio, vienen organizándose desde inicios de siglo, y fundan la Central Unitaria de Trabajadores (CUT) en 1953, con lo que logran representación institucional. Como concluye Bauer, los pactos que se fraguaban en la ciudad "a expensas de los trabajadores del campo", mantuvieron a este último "anclado al siglo diecinueve” (275). El gobierno de Ibáñez (1952-1958), en particular, está marcado por un hecho económico que anuncia el ocaso del sistema desarrollista: la creciente inflación provoca sucesivas huelgas generales en la década. En términos políticos, por otra parte, se vive una "democracia restringida", pues recién el último año de su gobierno el presidente derogará la Ley de Defensa Permanente de la Democracia, que proscribía al Partido Comunista, y que había sido firmada por el último gobernante radical en 1947.

Este es, pues, el primer escenario. Resulta interesante que Droguett escoja el campo y no la ciudad, por una parte, y el bandolerismo, por otra. Se decide no

\footnotetext{
${ }^{10}$ El epíteto hace referencia a un tipo de desarrollo de carácter tecnócrata, que tiene en el modelo económico de Industrialización por Sustitución de Importaciones (ISI) su cumbre. Este modelo nacionalista de bienestar social fue implementado por los gobiernos radicales (1938-1952).
} 
por el discurso ideológico programático de los partidos y su accionar en bloque, sino por una figura -el bandido rural- que "articula una respuesta agresiva, espontánea y sin ideología" (Salazar y Pinto 104). Diríase que la elección de Droguett es anacrónica, pues los bandidos rurales habían dejado de tener gravitancia como problemática nacional hacía décadas, y la acción política desde 1938 se había trasladado a la ciudad, hacia donde la población campesina no dejaba de trasladarse ${ }^{11}$. De ahí, sin embargo, la peculiaridad de este bandido rural que no está al acecho de la hacienda, sino que viene de la ciudad, acaso de sus extramuros, con lo que describe un tránsito inverso al de los sujetos históricos, y obliga a volver la vista sobre ese territorio cuyas prácticas y significado no estaba en la primera plana de las preocupaciones.

¿Y por qué bandolero? Si habíamos dicho que tal condición lo ubica "más acá de la duda o de las certezas políticas" (Concha 130), al mismo tiempo vincula a Eloy con una forma de rebeldía que escapa a cualquier acuerdo o negociación colectiva, que lo exime de hablar por ese colectivo. Ahora bien, el bandido rural es un tipo popular que ya se encuentra en la literatura criollista, y que comparte con las figuras del huaso y el roto características clave. A decir verdad, solo se diferencia de aquellos en su oficio, que incide en su construcción literaria únicamente para enfatizar su fuerza física y bravura. Esta configuración sintoniza con cierta mirada del bandido complaciente, romántica y en cualquier caso aristocrática, arraigada en una idea de heroicidad decimonónica ${ }^{12}$. Por esta razón, nos interesa leer al bandido de Droguett en contraste con esas otras dos figuras ${ }^{13}$.

\footnotetext{
${ }^{11}$ La misma historia del Ñato Eloy había ocurrido hacía más de una década, pues el bandido fue ajusticiado en 1941. Sobre la figura del bandido desde una perspectiva histórico-cultural, véase Daniel Palma Alvarado. Ladrones. Historia Social y Cultura del Robo en Chile, 1870-1920. Santiago de Chile: LOM, 2011 y Jaime Valenzuela Márquez. Bandidaje rural en Chile Central: Curicó, 1850-1900. Santiago de Chile: Dibam, 1991.

${ }^{12}$ Cfr. Lihn 14-24.

${ }^{13}$ En este sentido, sería interesante comparar la figura del bandido en Eloy y en Gran Señor y Rajadiablos (1948), novela del también chileno Eduardo Barrios. Para este autor, que narra desde el punto de vista de un latifundista heredero de los conquistadores españoles, el bandido es la peor amenaza al desarrollo de la hacienda, tomada como alegoría del Estado-nación. Véase Grínor
} 
En términos políticos, como señala Patrick Barr Melej, la narrativa criollista estaba vinculada con el Partido Radical, y había hecho del sujeto popular chileno un tipo fijo, pasivo y obediente: el huaso (79). A su vez, cuando recurría al roto para expresar la "chilenidad", lo anclaba al carácter heroico de que lo había revestido la Guerra del Pacífico ${ }^{14}$. Así, estos símbolos identitarios, encarnados en tipos populares (incluido el bandido), fueron utilizados por los criollistas con fines anti-oligárquicos, pues culpaban del subdesarrollo nacional a los oligarcas ${ }^{15}$. Como hemos señalado, una vez que inicia el desarrollismo, las capas medias (caldo de cultivo criollista) no dudaron en sostener alianzas con los terratenientes. El bandido, más cercano al roto, no es presentado por Droguett de modo maniqueo, como representante de contenidos dados de antemano; al contrario, le es restituido su carácter auténticamente rebelde y desestabilizador, es decir, su capacidad de expresar fuerzas que escapan a la vigilancia y neutralización política. En síntesis, Droguett resemantiza un arquetipo para derrumbar la construcción esencialista y hegemónica de la identidad chilena, y para problematizar una dinámica territorial.

Ahora bien, ¿cómo explicarse la edición definitiva de la novela en el año 1982? Esto dice relación con el segundo escenario de producción del texto. Como es sabido, con la Reforma Agraria la vida en los campos se politizará paulatinamente, para luego acelerarse en el gobierno de Salvador Allende (19701973) y ser cancelada de golpe con una contrarrevolución neoliberal a cargo de una dictadura militar (1973-1989). Desde nuestra perspectiva, no resulta tan

Rojo. Las Novelas de la Oligarquía Chilena. Santiago de Chile: Sangría, 2011. Es muy probable que Droguett haya leído esta novela, ampliamente difundida en la época.

${ }^{14}$ Por esos años, las figuras del huaso y del roto son representativas de la identidad chilena, que posee un sustrato racial -mestizaje- e ideológico-conservador-. Si bien el primer personaje fue el preferido por el criollismo debido a que encarnaba el ambiente rural, también dedicaron muchas páginas al segundo. En palabras de Subercaseaux, el huaso "se inscribe en la escenificación de un tiempo histórico nacional en clave de integración" (136). Del otro lado, históricamente, el roto corresponde a los peones que vagaban por el campo sin trabajo ni hogar fijos; el roto es una "figura híbrida de campo y urbe" (136).

${ }^{15}$ Barr-Melej sintetiza bien estas características del movimiento: "In essence, criollismo may be considered both antioligarchic for its delegitimization of high society's preeminence and antirevolutionary for its representation of labor" (78). 
importante la sindicalización campesina como el hecho de que la misma Reforma Agraria se haya presentado para el gobierno Demócrata-Cristiano como el destino bajo cuyo signo debía instalarse el país para alcanzar el desarrollo, a saber, al alero de la Alianza para el Progreso ${ }^{16}$. Este hecho solo puede ser interpretado como una ironía de la Historia, pues luego se amarró ese destino nacional de modo tanto más férreo a la voluntad del mismo gran hermano. En este contexto, adoptar el punto de vista de quien se halla al margen del Estado es acaso la única estrategia posible para problematizar el modelo apocalíptico de salvación y sostener una resistencia a un destino que de otro modo se pensaría como Destino Manifiesto. Es decir, podemos leer dos significaciones distintas del bandido: en este último caso, la terrible soledad de Eloy es tal vez demasiado certera a la hora de cancelar las redenciones sociales que la Historia ha levantado. En aquel sentido no biográfico es que interpretamos la frase de Droguett: “Eloy soy yo” (1996).

\title{
Bibliografía
}

\author{
Bauer, Arnold. La Sociedad rural chilena: desde la Conquista española a \\ nuestros Días. Santiago de Chile: Andrés Bello, 1994.
}

\footnotetext{
${ }^{16}$ Como apunta Bauer, para el Departamento de Estado norteamericano, Sobre la política externa norteamericana en la Guerra Fría: “en América Latina se necesitaba una 'reforma agraria', para cambiar las condiciones y reducir el presunto entusiasmo de los militantes marxistas por una insurrección rural" (272). En un interesante artículo, Sofía Correa ha establecido el nexo que existe entre la reestructuración neoliberal de la dictadura y la misión Klein-Saks, como se llamó a la consultora estadounidense que el Presidente Ibáñez contrató en 1955 para diagnosticar el problema inflacionario y sugerir cursos de acción que acabaran con el mismo. Las medidas tendían a liberalizar la economía y constituían, en palabras de Correa, "un programa global de reorganización de la economía chilena" (109). Digamos, entonces, que Eloy se edita por primera vez ad portas del antecedente directo del neoliberalismo en Chile, y por última vez ya durante la instalación sistemática de dicho régimen, a partir de los años ochenta.
} 
Barr-Melej, Patrick. "Introduction"; "Rewriting Chile. Criollismo and the Generation of 1900”. Reforming Chile: Cultural Politics, Nationalism and the Rise of the Middle Class. Chapel Hill: University of North Carolina Press, 2001. 1-17; 77-101.

Concha, Jaime. "En los Aledaños de El Compadre. Sufrimiento e Historia en Carlos Droguett". Coloquio internacional sobre la Obra de Carlos Droguett. Poitiers: Universidad de Poitiers, 1983. 105-138.

Correa, Sofía. "Algunos antecedentes históricos del proyecto neoliberal en Chile (1955-1958)". Opciones, n6 (1985): 106-146.

Droguett, Carlos. [1959]. Eloy. Santiago de Chile: Tajamar, 2008.

---. "Entrevista póstuma a Carlos Droguett: «Eloy soy yo»”. Entr. Leo Wetli, Punto Final, sept. 1996: 16-21.

---. [1940]. Los Asesinados del Seguro Obrero. Valparaíso: Ediciones Universitarias de Valparaíso, 1972.

Fink, Bruce. "Perversion”. Molly Anne Rothenberg, Denis Foster y Slavoj Zizek (eds.). Perversion and the Social Relation. Durham/London: Duke University Press, 2003. 38-67.

Girard, René. La Violencia y lo Sagrado. Trad. Joaquín Jordá. Barcelona: Anagrama, 2005.

Lihn, Enrique. "Los bandidos y el cuento chileno". En Enrique Lihn (Selección y prólogo). Diez Cuentos de Bandidos. Santiago de Chile: Quimantú, 1972. 525.

Lomelí, Francisco. La Novelística de Carlos Droguett: Poética de la Obsesión y el Martirio. Madrid: Playor, 1983.

Penney, James. The World of Perversion: Psychoanalysis and the Impossible Absolute of Desire. Albany: State University of New York Press, 2006.

Salazar, Gabriel y Pinto, Julio (eds.). Historia Contemporánea de Chile. Tomo II. Actores, identidad y movimiento. Santiago de Chile: LOM, 1999. 
Sicard, Alain. "Epílogo". Carlos Droguett. Eloy. Santiago de Chile: Ediciones Tajamar, 2008.

Subercaseaux, Bernardo. Historia de las ideas y de la cultura en Chile. Tomo IV. Nacionalismo y cultura. Santiago de Chile: Editorial Universitaria, 2007.

Zizek, Slavoj. "The Ambiguity of the Masochist Social Link". Molly Anne Rothenberg, Dennis Foster y Slavoj Zizek (eds.). Perversion and the Social Relation. Durham/London: Duke University Press, 2003. 112-125. 